\title{
1 ABSTRACT
}

\section{PURPOSE}

3 To determine whether combining training in heat with 'Live

4 High, Train Low' hypoxia (LHTL) further improves

5 thermoregulatory and cardiovascular responses to a heat

6 tolerance test compared to independent heat training.

\section{$7 \quad$ METHODS}

8 Twenty-five trained runners $\left(\mathrm{VO}_{2 \text { peak }}=64.1 \pm 8.0 \mathrm{ml} \cdot \mathrm{min} \cdot \mathrm{kg}^{-1}\right)$

9 completed three-weeks training in one of three conditions: 1)

10 Heat training combined with LHTL $\left(\mathrm{H}+\mathrm{H} ; \mathrm{F}_{\mathrm{i}} \mathrm{O}_{2}=14.4 \%(3000\right.$

$11 \mathrm{~m}), 13 \mathrm{~h} \cdot \mathrm{day}^{-1}$; train at $\left.<600 \mathrm{~m}, 33^{\circ} \mathrm{C}, 55 \% \mathrm{RH}\right)$; 2) heat

12 training (HOT; live and train $<600 \mathrm{~m}, 33^{\circ} \mathrm{C}, 55 \% \mathrm{RH}$ ); 3 )

13 temperate training (CONT; live and train $<600 \mathrm{~m}, 13^{\circ} \mathrm{C}, 55 \%$

$14 \mathrm{RH})$. Heat adaptations were determined from a 45 min heat

15 response test $\left(33^{\circ} \mathrm{C}, 55 \% \mathrm{RH}, 65 \% \mathrm{vVO}_{2 \text { peak }}\right)$ at baseline, 16 immediately, one and three weeks' post exposure (Baseline,

17 Post, 1wkP and $3 \mathrm{wkP}$, respectively). Core temperature, heart

18 rate, sweat rate and sodium concentration, plasma volume, and

19 perceptual responses were analysed using magnitude based

20 inferences.

\section{RESULTS}

Submaximal heart rate $(E S=-0.60(-0.89 ;-0.32))$ and core temperature $[\mathrm{ES}=-0.55(-0.99 ;-0.10)]$ were reduced in HOT until $1 w k P$. Sweat rate $[E S=0.36(0.12 ; 0.59)]$ and sweat sodium concentration $[\mathrm{ES}=-0.82(-1.48 ;-0.16)]$ were respectively increased and decreased until $3 \mathrm{wkP}$ in HOT. Submaximal heart rate $[E S=-0.38(-0.85 ; 0.08)]$ was likely reduced in $\mathrm{H}+\mathrm{H}$ at $3 \mathrm{wkP}$, whilst $\mathrm{CONT}$ had unclear physiological changes. Perceived exertion and thermal sensation were reduced across all groups.

\section{CONCLUSIONS}

Despite greater physiological stress from combined heat training and LHTL, thermoregulatory adaptations are limited in comparison to independent heat training. The combined stimuli provides no additional physiological benefit during exercise in hot environments.

KEYWORDS: altitude, cross-tolerance, endurance, acclimation, environment 
INTRODUCTION

Exercise in environments such as hypoxia or heat acutely increases physiological strain and reduces performance capacity $^{1-3}$. Repeated exposure to hypoxia drives haematological and muscular adaptations to improve aerobic capacity in both hypoxic and normoxic environments ${ }^{4}$. Heat training and acclimation reduces thermal and cardiovascular strain during exercise; predominantly, via reduced core temperature, increased plasma volume (PV), increased sweat rate and earlier sweat onset ${ }^{3}$. The benefits of both heat and hypoxia can last for several weeks following exposure ${ }^{6,7}$. As heat and hypoxia have similar adaptive response pathways ${ }^{8}$, investigators have recently explored the potential additive effect of combining heat and hypoxia to enlarge physiological adaptations and delay the decay of these responses ${ }^{2,9}$.

An initial study in team sport athletes combined heat training and 'Live High, Train Low' (LHTL) hypoxia during a preseason camp, and reported a sustained increase in PV and haemoglobin mass $\left(\mathrm{Hb}_{\text {mass }}\right)$ compared to heat training alone ${ }^{9}$. Conversely, a recent study in endurance athletes demonstrated that adding LHTL to isothermally controlled heat acclimation had no additional physiological benefit ${ }^{2}$. However, the hypoxic dose supplied was reduced compared to previous LHTL studies reporting physiological and performance benefits in endurance athletes 10,11. Therefore, the resulting physiological responses to combined heat training with LHTL hypoxia in endurance athletes is relatively unknown.

The positive interactions between heat and hypoxia have been suggested to result from the activation of similar cellular protective pathways, with heat shock proteins (Hsp) and hypoxic-inducible factor $1 \alpha$ (HIF-1 $\alpha$ ) proposed to be key metabolic links ${ }^{8}$. The highly inducible Hsp70/72 family are elevated immediately following acute heat or hypoxic exposure ${ }^{12}$, as well as periods of heat acclimation ${ }^{13}$, and assist in the stabilisation of HIF- $1 \alpha^{8}$ during cellular stress. Activating the HIF-1 $\alpha$ pathway signals the release of erythropoietin and vascular endothelial growth factor (VEGF) to promote angiogenesis, increasing muscle oxygen delivery in hypoxia and potentially increasing skin blood flow in hot environments ${ }^{8}$. However, not all responses are similar, with heat exposure eliciting hemodilution effects, and hypoxia promoting hemoconcentration ${ }^{14}$. Research these interactive heat and hypoxic acclimation pathways in endurance athletes is currently limited. 
The potential physiological benefits of combined heat training and LHTL warrants further research. Specifically, the potential physiological outcomes during exercise in a hot environment. Therefore, this study aimed to investigate the appearance and decay of thermal, cardiovascular and biochemical responses in endurance athletes following three weeks of heat training with or without LHTL, compared to temperate training alone. It was hypothesised that heat training would reduce heat strain during a heat response test, and LHTL would enhance thermoregulatory and cardiovascular responses, and have less decay in the following weeks.

\section{METHODS}

\section{Experimental Overview}

This study incorporated a multicentre, parallel, matched group experimental design, as part of a larger project investigating the effects of combined heat and hypoxia on temperate performance in trained runners ${ }^{15}$. Twenty-five trained male and female runners were assigned into one of three groups: 1) Heat training plus LHTL hypoxia $(\mathrm{H}+\mathrm{H})$; 2) heat training with no hypoxic exposure (HOT); or 3) temperate training only (CONT) (Figure 1). Baseline characteristics are presented in Table 1, with participants matched on prior training load, peak oxygen uptake $\left(\mathrm{VO}_{2 \text { peak }}\right)$ and associated velocity ( $\left.\mathrm{vVO}_{2 \text { peak }}\right)$, and then randomly assigned to groups (coin toss/number) based on geographic location by an independent associate. Participants completed a three-week training period, incorporating $3 \times 90$ min treadmill sessions per week in their allocated environmental conditions, followed by three-weeks living and training in normoxic, temperate conditions. Testing was conducted prior, immediately post, one week and three weeks following the exposure period (Baseline, Post, 1wkP and $3 w k P$, respectively).

\section{INSERT FIGURE 1 HERE \\ INSERT TABLE 1 HERE}

Participants had $\geq 2$ y competitive running experience and regularly completed 10-20 h of weekly training. An additional three participants were not included in the analysis due to illness $(n=1)$ and incomplete fulfilment of testing requirements $(n=2)$. Due to logistical constraints, and to minimise the loss of heat acclimation benefits, menstrual cycle was recorded but not controlled. All groups commenced with a mixture of menstrual phases $(\mathrm{H}+\mathrm{H}$ : luteal $(\mathrm{n}=1)$, follicular $(\mathrm{n}=1)$, not menstruating $(n=2)$; HOT: luteal $(n=2)$, follicular $(n=1)$; CONT: luteal $(n=1)$, follicular $(n=1)$ ). Considering the endurance-trained status of the female athletes, the mix of menstrual phases was 
anticipated to have minimal impact on heat acclimation responses ${ }^{16}$. No participant had any heat or hypoxic exposure during the four weeks prior, and all training and testing was conducted during the winter and spring months. Prior to the study, participants were informed of all procedures and potential risks involved in the study and a written informed consent was obtained. The study was approved by the Ethics Committee of the University of Technology Sydney (Trial no UTS HREC 2014000203).

\section{Training Details}

A normobaric hypoxic facility at the Australian Institute of Sport (AIS) was utilised for LHTL exposure. Heat sessions were completed in a climatic chamber (Altitude Training Systems, Lidcombe, Australia) at either the University of Canberra or the New South Wales Institute of Sport (NSWIS) in Sydney. The CONT group completed treadmill sessions in temperate conditions. Environmental details and training sessions are outlined in Figure 1. To replicate the demands of an athletes' typical training program, training intensity was matched to individual $\mathrm{vVO}_{2 \text { peak, }}$ as determined at Baseline in temperate, normoxic conditions. To maintain previous training load, participants completed additional low intensity aerobic training in normoxic, temperate conditions throughout the study. Training load (AU) for all sessions was monitored using the session rating of perceived exertion (sRPE) method, calculated as the product of training duration (min) and the mean training intensity (RPE, CR-10) ${ }^{17}$.

\section{Incremental treadmill test}

An initial incremental test was completed on a calibrated motorised treadmill for assessment of $\mathrm{VO}_{2 \text { peak }}$ and $\mathrm{vVO}_{2 \text { peak }}$ (Canberra; custom-built motorised treadmill, AIS. NSWIS: Payne Treadmill, Stanton Engineering, Girraween, Australia). Briefly, starting speed was increased by $1 \mathrm{~km} \cdot \mathrm{h}^{-1}$ each minute for 4 min, after which gradient was increased $1 \%$ every minute until volitional exhaustion was reached. Heart rate (HR; Suunto T6, Vantaa, Finland) and oxygen consumption (Canberra: in-house automated metabolic system; NSWIS: Moxus Modular Metabolic System, AEI Technologies, Pittsburgh, USA) were measured continuously and averaged into $30 \mathrm{~s}$ periods for analysis.

\section{Heat Response Test}

The heat response test involved a 45 min treadmill run $\left(33^{\circ} \mathrm{C}\right.$, $\left.55 \% \mathrm{RH}, 65 \% \mathrm{vVO}_{2 \text { peak }}\right)$, followed by $30 \mathrm{~min}$ passive recovery and was completed as session one (Baseline), session nine (Post), as well as $1 \mathrm{wkP}$ and $3 \mathrm{wkP}$. To allow a direct comparison to Baseline, there was no adjustment to intensity 
across the testing sessions and tests were completed at the same location and a similar time of day. Upon arrival, participants rested in a supine position for $20 \mathrm{~min}$ in a temperate environment $\left(21^{\circ} \mathrm{C}\right)$, then gave a blood sample from the antecubital vein. Participants provided a urine sample to determine urine specific gravity (UG1, Atago Co., Ltd, Tokyo, Japan) and osmolality (Model 3250 Osmometer, Advanced Instruments Inc, Norward, USA). A pre-test urine osmolality below $700 \mathrm{osmol} \cdot \mathrm{kg}^{-1}$ and urine specific gravity below 1.020 was considered a euhydrated state ${ }^{18}$. Participants' drank water ad libitum until test commencement. No fluid was consumed during the test, with pre- and post-body mass measured in minimal clothing for estimation of sweat rate (Digi DI-160, Wedderburn, Ingleburn, Australia). An adhesive sweat patch (Tegaderm+ Pad, 3M Health Care, Borken, Germany) was attached to the upper side of the right scapular, and analysed for sweat sodium concentration ([Na $]_{\text {sweat }}$; Cobras 400 Plus, Roche Diagnostics Ltd, Rotkreuz, Switzerland). Heart rate (HR, Suunto T6, Vantaa, Finland) and core temperature (Squirrel Data logger, Grant Instruments, Cambridge, UK) were recorded continuously, with core temperature measured via a temperature probe (Mon-a-therm, Mansfield, USA) inserted 10 $\mathrm{cm}$ beyond the anal sphincter. Skin temperature was recorded in one minute averages via thermal sensors (Thermochron iButton, Maxim Integrated, San Jose, USA) attached to four different sites (chest, forearm, thigh, calf). The weighted mean skin temperature was calculated according to Ramanathan ${ }^{19}$. Core temperature, heart rate and skin temperature were analysed as mean values during exercise. Perceptual measures of thermal sensation ${ }^{20}$, and a rating of perceived exertion (RPE, CR-10) ${ }^{21}$ were assessed every $10 \mathrm{~min}$ and at the conclusion of exercise, and combined into a mean value for analysis.

\section{Blood Biochemistry}

A venous blood sample was taken from the participant's antecubital vein in the three weeks prior to study commencement for blood ferritin assessment (Vacuette ${ }$, Greiner Bio-One, Frickenhausen, Germany). Collected samples were centrifuged at $3000 \mathrm{rpm}, 4^{\circ} \mathrm{C}$, for $10 \mathrm{~min}(2-16 \mathrm{~K}$, Sigma Laborzentrifugen GmbH, Osterode am Harz, Germany), and transported for same day commercial biochemical analysis (NSWIS: Douglass Hanly Moir Pathology, Macquarie Park, Australia; Canberra: AIS Biochemisty Laboratory). Participants with levels $<100 \mathrm{ug} \cdot \mathrm{L}^{-1}$ were provided a daily oral iron supplement for the study duration (Ferrograd C, $325 \mathrm{mg}$ dried ferrous sulphate $+562.4 \mathrm{mg}$ sodium ascorbate; Abbott, Botany, Australia). 
Resting venous blood samples were taken prior to each heat response test for determination of Hsp70 and VEGF. Samples were centrifuged, separated into $500 \mu \mathrm{L}$ plasma aliquots, and stored at $-80^{\circ} \mathrm{C}$ for later analysis via enzyme-linked immunosorbent assay (ELISA) kits according to manufacturer's instructions (Hsp70: ADI-ESK-715, Enzo Life Sciences Inc., Farmingdate, USA, CV = 7.1\%; VEGF: DVE00, R\&D Systems Inc., Minneapolis, USA, CV = 5.4\%). Assays were conducted on a SpectraMax 190 microplate reader (Molecular Devices LLC, Sunnyvale, USA). Prior to analysis, measures were adjusted for plasma volume differences ${ }^{22}$.

\section{Plasma and Blood Volume}

Plasma volume (PV) and blood volume (BV) were indirectly calculated by the optimized CO rebreathing procedure (OSM3, Radiometer, Copenhagen, Denmark) ${ }^{23}$. Haemoglobin mass $\left(\mathrm{H} b_{\text {mass }}\right)$ was additionally measured, with detailed methods and results previously reported ${ }^{15}$. Baseline values were averaged into a single time point for analysis, with the typical error of measurement (TE) for PV calculated at 3.6\% (2.8-4.8\%, 90\% confidence limits).

\section{Statistical Analysis}

Data was assessed according to magnitude based-inferences ${ }^{24}$. Data were log-transformed for analyses, to reduce bias from any non-uniformity of error, and back-transformed to obtain changes in means and variation as percent. Data are presented as means with $90 \%$ confidence limits (CL) unless otherwise stated. Mean percent change $( \pm 90 \% \mathrm{CL})$ for variables were calculated as the difference from $\mathrm{H}+\mathrm{H}$ and $\mathrm{HOT}$ compared to CONT. Effects were deemed unclear if the confidence interval overlapped the thresholds for the smallest positive and negative effects, with clear effects assessed as following: > 25-75\%, possible; > 75-95\%, likely; >95-99\%, very likely; > 99\%, almost certain. The smallest worthwhile change was calculated as a standardised small effect size $(\mathrm{ES}=0.2)$ multiplied by the pre-test between-subject standard deviation (SD) ${ }^{25}$. Typical error of measurement for outcome measures were calculated from the SD of the change scores divided by the mean and presented as a coefficient of variation $(\%)$.

\section{RESULTS}

\section{Environmental Exposure and Training Load}

Both HOT and $\mathrm{H}+\mathrm{H}$ received $13.5 \mathrm{~h}$ total heat during the threeweek exposure period, with CONT receiving $2.5 \mathrm{~h}$. All groups received $2.5 \mathrm{~h}$ heat during the following three weeks from 
subsequent heat response tests. Specific training load information is described elsewhere ${ }^{15}$, however it should be noted that there were no clear training load differences between groups across the six-week study duration.

\section{Heat Response Test}

\section{Physiological Measures}

All comparative changes are relative to Baseline. Heart rate was most likely reduced in HOT at Post, however was unclear by $3 w k P$ (Figure 2). There was a possible HR decrease at Post in $\mathrm{H}+\mathrm{H}$, and a likely reduction at $3 \mathrm{wkP}$. Table 2 shows that HR was most likely and likely reduced at Post in HOT when compared to CONT and $\mathrm{H}+\mathrm{H}$, respectively. This difference was also evident $1 \mathrm{wkP}$ (CONT: very likely, $\mathrm{H}+\mathrm{H}$ : likely), but became unclear by $3 \mathrm{wkP}$.

Within group measures are outlined in Table 2, and between group comparisons are displayed in Figure 3. Of note, core temperature was lowered in HOT at Post, remaining likely reduced at $1 \mathrm{wkP}$ but unclear at $3 \mathrm{wkP}$. Sweat rate was likely increased in HOT at $1 \mathrm{wkP}$ and $3 \mathrm{wkP}$. Skin temperature was likely increased in $\mathrm{H}+\mathrm{H}$ at Post and most likely higher at $3 \mathrm{wkP}$, while HOT was possibly reduced at Post and $1 \mathrm{wkP}$. There was a likely decrease in $[\mathrm{Na}]_{\text {sweat }}$ in HOT across each time point. $[\mathrm{Na}]_{\text {sweat }}$ in $\mathrm{H}+\mathrm{H}$ was very likely lowered at Post, remaining possibly reduced at $1 \mathrm{wkP}$ and $3 \mathrm{wkP}$.

\section{INSERT TABLE 2 AND FIGURE 3 HERE}

\section{Perceptual Measures}

RPE was likely and very likely reduced in $\mathrm{H}+\mathrm{H}$ and HOT respectively at Post and $1 \mathrm{wkP}$, and further reduced at $3 \mathrm{wkP}$ in both groups (Table 2). Despite CONT being unclear at Post, RPE was reduced at $1 \mathrm{wkP}$ and $3 \mathrm{wkP}$. Thermal sensation decreased at each time point in HOT and $\mathrm{H}+\mathrm{H}$, while CONT had reduced thermal sensation at $1 \mathrm{wkP}$ only. The $\mathrm{H}+\mathrm{H}$ group had a greater reduction Post compared to both HOT and CONT. 
Plasma volume was possibly increased by $3.8 \pm 6.0 \%$ in HOT $[E S=0.13(-0.07 ; 0.34)]$ from Baseline to Post, which was possibly higher when compared to $\mathrm{H}+\mathrm{H}[\mathrm{ES}=0.23(-0.08$; $0.54)]$ and CONT $[\mathrm{ES}=0.17(-0.13 ; 0.47)]$. At $1 \mathrm{wkP}, \mathrm{HOT}$ remained possibly greater than $\mathrm{H}+\mathrm{H}[\mathrm{ES}=0.22(-0.05 ; 0.50)]$, but at $3 w k P$ there were no differences between any groups. A small BV increase in HOT produced a possibly greater increase compared to $\mathrm{H}+\mathrm{H}$ by $3.7 \pm 5.4 \%$ at Post $[\mathrm{ES}=0.14(-0.06$; 0.35)].

\section{Hsp70 and VEGF}

The $\mathrm{H}+\mathrm{H}$ group had a likely decrease in Hsp70 from Post to $3 w k P[E S=-0.48(-0.87 ;-0.09)]$, resulting in a likely reduction at $3 \mathrm{wkP}$ relative to Baseline $[\mathrm{ES}=-0.42(-0.91 ; 0.06)]$. Changes were unclear in HOT and CONT, with no clear between group differences. All between and within group changes in VEGF were unclear or likely trivial.

\section{DISCUSSION}

Despite $\mathrm{HOT}$ and $\mathrm{H}+\mathrm{H}$ receiving the same exposure to heat, the 344 addition of LHTL to heat training negated some thermoregulatory adaptations during submaximal running in a hot environment. HOT elicited cardiovascular and thermal adaptations, including a reduction in submaximal HR, core and skin temperature. Sweat responses in HOT were enhanced up to $3 \mathrm{wkP}$ through changes to sweat rate and $[\mathrm{Na}]_{\text {sweat }}$, supporting the concept that the slowest appearing adaptations also have the slowest decay ${ }^{6}$. In support of our hypothesis, incorporating nine heat interval-training sessions across three-weeks sufficiently elicits heat acclimation adaptations and reduced heat strain during submaximal exercise in the heat.

We hypothesised that the greater physiological strain from combined heat and LHTL, would accelerate the cardiovascular and thermoregulatory responses during a heat response test. In contrast to our hypothesis, heat training and LHTL elicited no changes in core temperature, PV or sweat rate. These findings differ to previous studies reporting similar heat adaptations following approximately 2 weeks of heat training or heat training combined with LHTL $^{2,9}$. The different findings may relate to factors including participant training status and environmental dose. Buchheit et al., ${ }^{9}$ assessed team sport athletes in an early season training camp with a lower endurance training status compared to the current participants. 
However, Rendell et al., ${ }^{2}$ examined well-trained endurance athletes, therefore the difference in our findings cannot be solely attributed to training status. Alternatively, the total heat and hypoxic dose (13.5 h heat, $293 \mathrm{~h}$ hypoxia) may explain the differing results, with previous studies having a higher ratio of heat to hypoxic exposure; ie. 26.5 heat and $170 \mathrm{~h}$ hypoxia $(\sim 3000 \mathrm{~m})$ over two weeks ${ }^{9}, 15 \mathrm{~h}$ heat and $100 \mathrm{~h}$ hypoxia $(2400 \mathrm{~m})$ over 11 days ${ }^{2}$. Another key difference between studies was the use of daily heat exposure in the previous studies, compared to an intermittent protocol in our study. In the present study, heat exposure was limited to three sessions per week, and training sessions were conducted at a fixed intensity rather than a fixed thermal load. Heat acclimation is suggested to be optimised with daily exposure and controlled thermal load ${ }^{3}$. The prescription from temperate training intensity, combined with the intermittent heat exposure was designed to represent a practical training design that could be implement by coaches and athletes, utilising training prescription methods that are routinely incorporated into their training routine. Nonetheless, the intermittent protocol utilised in the present study was adequate to elicit heat responses in the HOT group. Considering the thermoregulatory responses were more prominent in the HOT group only, it is plausible the heat dose was not sufficient to overcome the hemoconcentration effects of the LHTL dose in the present study ${ }^{14}$. For example, $\mathrm{PV}$ did not change in $\mathrm{H}+\mathrm{H}$, compared to $+6 \%$ increase in previous studies ${ }^{2,9}$, indicating the current heat dose was only adequate to neutralise any associated hemoconcentration. Based on these observations, we suggest that if incorporating a large hypoxic dose into a heat-training block, an adjustment of heat exposure may be required to elicit complete thermoregulatory adaptations. However, caution must be taken in regards to the overall stress applied to the athlete using this combined approach.

In contrast to previous reports ${ }^{8}$, our findings do not support the concept of heat and hypoxic cross-acclimation enhancing thermoregulatory adaptations during exercise in the heat. While $\mathrm{H}+\mathrm{H}$ and HOT both demonstrated $[\mathrm{Na}]_{\text {sweat }}$ conservation, sweat rate increases were only observed in the HOT group. Furthermore, skin temperature increased in $\mathrm{H}+\mathrm{H}$, indicating a reduction of heat dissipation. These findings agree with Minson et al., ${ }^{26}$ who reported acute hypoxia increases blood flow competition between the skin and splanchnic areas, resulting in reduced skin blood flow and sweat rate for a given core temperature. Interestingly, $\mathrm{H}+\mathrm{H}$ elicited $[\mathrm{Na}]_{\text {sweat }}$ conservation, which is largely controlled during exercise by secretion of the hormone aldosterone from the sweat glands and kidneys ${ }^{27}$. While hypoxia is suggested to initially decrease 
aldosterone, levels have been reported to return to sea level concentrations after 12-20 days living in an hypoxic environment ${ }^{28}$. Given the 21 days of exposure in the present study, this may explain the $[\mathrm{Na}]_{\text {sweat }}$ conservation despite no sweat rate changes. However, with no direct measure of aldosterone, the impact of prolonged hypoxic exposure on sweat responses and thermoregulation during heat exposure warrants further investigation.

Whilst it has been suggested that VEGF activation through the HIF-1 $\alpha$ pathway is a key contributor between heat and altitude adaptations ${ }^{8}$, there were no VEGF changes in any group in the present study. Similarly, Hsp70 was unchanged in the HOT group throughout the study period. Increases in extracellular Hsp72 have been reported after moderate and high intensity exercise in the heat ${ }^{12}$, however no consensus exists on basal plasma Hsp70 responses following either heat or hypoxia ${ }^{29}$. A possible explanation for these observations may be due to the intermittent nature of the heat exposure in the current study, with Gibson et al., ${ }^{30}$ demonstrating post-exercise increases in plasma Hsp72 returning to baseline by $24 \mathrm{~h}$ post. However, the high individual variability of VEGF and Hsp70, combined with the relatively poor sensitivity of the ELISA kits, cannot be discounted as explanations for the few changes in these variables. There was an unexpected reduction in Hsp70 at $3 \mathrm{wkP}$ in $\mathrm{H}+\mathrm{H}$. Whilst evidence is limited, lower extracellular Hsp70/72 following heat acclimation has been attributed to a reduction in cellular stress following removal of the heat stressor ${ }^{29}$. It is possible that the combined heat and hypoxia provides greater cellular stress, resulting in an increased reduction in cellular stress and Hsp70 requirements in the weeks following exposure. However, with no other changes to Hsp70, we are unable to provide a clear mechanistic reasoning behind the reduction at $3 \mathrm{wkP}$.

Despite different thermoregulatory responses in each experimental group, thermal comfort and RPE were reduced across all groups. The greater reduction in RPE and thermal comfort in $\mathrm{H}+\mathrm{H}$ compared to HOT shows an uncoupling of physiology and perception. Notably, despite the heat response tests being spread across six-weeks to minimize heat responses in CONT ${ }^{31}$, RPE and thermal comfort were reduced at $1 \mathrm{wkP}$ in CONT. This indicates that the short duration between Post and $1 \mathrm{wkP}$ tests produced some perceptual adaptations. These findings further highlight that a better understanding of athletes' response to training stress occurs when a myriad of perceptual and physiological measures are taken ${ }^{32}$. 
The influence of individual responses to both independent heat $^{33}$ and hypoxia ${ }^{34}$ can have a limiting influence on the overall results, particularly with small group sizes. The authors acknowledge the influence of individual results on the findings, and while steps were taken to minimise the influence of external factors such as iron status and training intensity on individual responses ${ }^{34}$, further research is required to assess the overall influence of individual responses. It should also be noted that blood ferritin assessment occurred only prior to study commencement, and as a result the authors can only assume based off previous research that iron absorption occurred in those athletes given iron supplementation ${ }^{35}$.

\section{PRACTICAL APPLICATIONS}

\section{CONCLUSION}

This study illustrates that independent heat training produces different physiological adaptations to exercise in the heat, compared to combined heat training and LHTL hypoxia. Core temperature, submaximal $\mathrm{HR}$ and sweat responses were impaired in the combined heat and LHTL group. Further investigations are required to assess of cross-acclimation benefits are present with a greater heat and/or lowered hypoxic 
LHTL adaptive responses needs to be assessed in order to provide a greater understanding for coaches and sport scientists implementing environmental stimuli into training programs.

\section{ACKNOWLEDGMENTS}

The authors wish to thank the Australian Institute of Sport and Australian Sports Commissions Big Ideas Fund for their financial support, Douglass Hanly Moir for blood analysis, and particular thanks to Chris Abbiss from Edith Cowan University for assistance with the assay analyses. Thanks to New South Wales Institute of Sport, University of Canberra Research Institute for Sport and Exercise, University of Technology Sydney and the AIS Department of Physiology. The authors have no conflicts of interest to declare.

\section{REFERENCES}

1. Wehrlin JP, Hallén J. Linear decrease in VO2max and performance with increasing altitude in endurance athletes. Eur J Appl Physiol. March 01 2006;96(4):404-412.

2. Rendell RA, Prout J, Costello JT, et al. Effects of 10 days of separate heat and hypoxic exposure on heat acclimation and temperate exercise performance. Am J Physiol Regul Integr Comp Physiol. 2017;313(3):R191-R201.

3. Tyler CJ, Reeve T, Hodges GJ, Cheung SS. The effects of heat adaptation on physiology, perception and exercise performance in the heat: A meta-analysis. Sports Med. Nov 2016;46(11):1699-1724.

4. Levine BD, Stray-Gundersen J. "Living high-training low": effect of moderate-altitude acclimatization with low-altitude training on performance. J Appl Physiol. July 1, 1997 1997;83(1):102-112.

5. Lorenzo S, Halliwill JR, Sawka MN, Minson CT. Heat acclimation improves exercise performance. J Appl Physiol.

6. Daanen HAM, Racinais S, Périard JD. Heat acclimation decay and re-induction: A systematic review and meta-analysis. Sports Med. November 112017.

7. Chapman RF, Laymon Stickford AS, Lundby C, Levine BD. Timing of return from altitude training for optimal sea level performance. J Appl Physiol. 2014-04-01 00:00:00 2014;116(7):837-843.

8. Ely BR, Lovering AT, Horowitz M, Minson CT. Heat acclimation and cross tolerance to hypoxia. Temperature. 2014/07/01 2014;1(2):107-114.

9. Buchheit $\mathrm{M}$, Racinais $\mathrm{S}$, Bilsborough J, et al. Adding heat to the live-high train-low altitude model: a practical insight from professional football. Br J Sports Med. December 1, 2013 2013;47(Suppl 1):i59-i69. 
10. Brugniaux JV, Schmitt L, Robach P, et al. Eighteen days of "living high, training low" stimulate erythropoiesis and enhance aerobic performance in elite middle-distance runners. J Appl Physiol. 2006;100(1):203-211.

11. Gore CJ, Hahn AG, Aughey RJ, et al. Live high:train low increases muscle buffer capacity and submaximal cycling efficiency. Acta Physiol Scand. 2001;173(3):275-286.

12. Périard JD, Ruell $P$, Caillaud $C$, Thompson MW. Plasma Hsp72 (HSPA1A) and Hsp27 (HSPB1) expression under heat stress: influence of exercise intensity. Cell Stress Chaperones. 2012;17(3):375-383.

13. Sandström ME, Siegler JC, Lovell RJ, Madden LA, McNaughton L. The effect of 15 consecutive days of heatexercise acclimation on heat shock protein 70. Cell Stress Chaperones. 2008;13(2):169-175.

14. Sawka MN, Concertino VA, Eichner ER, Schnieder SM, Young AJ. Blood volume: importance and adaptations to exercise training, environmental stresses, and trauma/sickness. Med Sci Sports Exerc. 2000;32(2):332.

15. McCleave EL, Slattery KM, Duffield R, et al. Temperate performance benefits after heat, but not combined heat and hypoxic training. Med Sci Sports Exerc. Mar 2017;49(3):509517.

16. Kuwahara $T$, Inoue $Y$, Abe $M$, Sato $Y$, Kondo N. Effects of menstrual cycle and physical training on heat loss responses during dynamic exercise at moderate intensity in a temperate environment. Am J Physiol Regul Integr Comp Physiol. May 2005;288(5):R1347-1353.

17. Foster C, Florhaug JA, Franklin J, et al. A new approach to monitoring exercise training. J Strength Cond Res. Feb 2001;15(1):109-115.

18. Sawka MN, Burke LM, Eichner ER, Maughan RJ, Montain SJ, Stachenfeld NS. American College of Sports Medicine position stand. Exercise and fluid replacement. Med Sci Sports Exerc. Feb 2007;39(2):377-390.

19. Ramanathan NL. A new weighting system for mean surface temperature of the human body. J Appl Physiol. May 1964;19:531-533.

20. Gagge AP, Stolwijk JA, Saltin B. Comfort and thermal sensations and associated physiological responses during exercise at various ambient temperatures. Environ Res. Apr 1969;2(3):209-229.

21. Borg GAV. Psychophysical bases of perceived exertion. Med Sci Sports Exerc. 1982;14(5):377-381.

22. Kargotich S, Goodman C, Keast D, Morton AR. The influence of exercise-induced plasma volume changes on the interpretation of biochemical parameters used for monitoring exercise, training and sport. Sports Med. 1998;26(2):101-117. 
23. Schmidt W, Prommer N. The optimised CO-rebreathing method: a new tool to determine total haemoglobin mass routinely. Eur J Appl Physiol. Dec 2005;95(5-6):486-495.

24. Batterham AM, Hopkins WG. Making meaningful inferences about magnitudes. Int J Sports Physiol Perform. 2006;1(1):50-57.

25. Cohen J. Statistical power analysis for the behavioral sciences 2 nd edition ed. Hillsdale, NJ: Lawrence Erlbaum Associates; 1988.

26. Minson CT. Hypoxic Regulation of Blood Flow in Humans. In: Roach RC, Wagner PD, Hackett PH, eds. Hypoxia: Through the Lifecycle. Boston, MA: Springer US; 2003:249-262.

27. Brandenberger $G$, Candas V, Follenius M, Libert JP, Kahn JM. Vascular fluid shifts and endocrine responses to exercise in the heat. Europ J Appl Physiol Occup Physiol. 1986;55(2):123-129.

28. Milledge JS, Catley DM, Ward MP, Williams ES, Clarke CR. Renin-aldosterone and angiotensin-converting enzyme during prolonged altitude exposure. J Appl Physiol Respir Environ Exerc Physiol. Sep 1983;55(3):699-702.

29. Magalhães FdC, Amorim FT, Passos RLF, et al. Heat and exercise acclimation increases intracellular levels of $\mathrm{Hsp72}$ and inhibits exercise-induced increase in intracellular and plasma Hsp72 in humans. Cell Stress Chaperones. 2010;15(6):885-895.

30. Gibson OR, Dennis A, Parfitt T, Taylor L, Watt PW, Maxwell NS. Extracellular Hsp72 concentration relates to a minimum endogenous criteria during acute exercise-heat exposure. Cell Stress Chaperones. 2014;19(3):389-400.

31. Barnett A, Maughan RJ. Response of unacclimatized males to repeated weekly bouts of exercise in the heat. BrJ Sports Med. 1993;27(1):39-44.

32. Le Meur Y, Hausswirth C, Natta F, Couturier A, Bignet F, Vidal PP. A multidisciplinary approach to overreaching detection in endurance trained athletes. J Appl Physiol. Feb 2013;114(3):411-420.

33. Racinais $\mathrm{S}$, Mohr M, Buchheit $\mathrm{M}$, et al. Individual responses to short-term heat acclimatisation as predictors of football performance in a hot, dry environment. Br J Sports Med. Sep 2012;46(11):810 - 815.

34. Sinex JA, Chapman RF. Hypoxic training methods for improving endurance exercise performance. J Sport Health Sci. 12// 2015;4(4):325-332.

35. Garvican LA, Saunders PU, Cardoso T, et al. Intravenous iron supplementation in distance runners with low or suboptimal ferritin. Med Sci Sports Exerc. 2014/02// 2014;46(2):376385. 
656

657

658

659

660

661

662

663

664

665

666

667

668 Figure 1. Experimental design

669

670

671

672

673

674

675

676

677

678

679

680 
685

686

687

688

689

690

691

692

693

694

695

696

697 Figure 2. Changes in average heart rate during the heat 698

699 response test, expressed as a percent change (\%) from Baseline $\pm 90 \%$ CL for $\mathrm{H}+\mathrm{H}(\mathrm{A})$, HOT (B), and CONT

700 (C). *Likely within group difference from Baseline.

701

702

703

704

705

706

707

708

709

710 
Figure 3. Comparison of the physiological responses between interventions, expressed as the standardised difference in the change for HOT v $\mathrm{H}+\mathrm{H}, \mathrm{H}+\mathrm{H} v \mathrm{CONT}$, HOT v CONT. Responses include core temperature (Core Temp), skin temperature (Skin Temp), sweat sodium concentration $\left([\mathrm{Na}]_{\text {sweat }}\right)$ and sweat rate during the heat response test. 